\title{
Dimensiones del pensamiento sistémico aplicado: un estudio de casos múltiple desde la perspectiva de sistemas complejos y el aprendizaje organizacional
}

Dimensões do pensamento sistêmico aplicado: um estudo de caso múltiplo na perspectiva de sistemas complexos e aprendizagem organizacional

\section{Dimensions of applied systemic thinking: a multiple case study from the complex systems and organizational learning perspective}

Gustavo Montenegro

ORCID ID: 0000-0002-2684-9550 Universidad Católica de Córdoba, Argentina

Ivana Schroeder

ORCID ID: 0000-0002-7304-902X Universidad Católica de Córdoba, Argentina

Autor referente: 0216373@ucc.edu.ar

Historia editorial Recibido: 31/08/2019 Aceptado: 02/06/2020

\section{RESUMEN}

Este artículo se desarrolla en el marco perspectivas de sistemas complejos y de una investigación interesada por las condiciones que intervienen en el desarrollo de aprendizaje en las organizaciones contemporáneas. Con apoyo en fundamentos teóricos del aprendizaje organizacional y un diseño metodológico de estudio de casos múltiple, el trabajo pone en foco el papel del pensamiento sistémico como condición relevante para el aprendizaje organizacional, observado en el marco de procesos de tratamiento 
y solución de problemas en pequeñas y medianas organizaciones de la ciudad de Córdoba (Argentina). Como resultados se presentan cuatro dimensiones relevantes de observar en torno al pensamiento sistémico en circunstancias de práctica concreta, y la hipótesis de que el pensamiento sistémico puede presentarse a modo de patrón autoorganizado. Entre las conclusiones se sostiene que el pensamiento sistémico incide de manera significativa en los procesos de tratamiento de problemas, $y$ consecuentemente en el logro de mejoras, aprendizajes compartidos, innovaciones y construcción de conocimiento en las organizaciones estudiadas, y que se manifiesta como emergente interaccional y contextual más que como competencia meramente individual. Los hallazgos tienen implicancias para investigadores, para gestores institucionales y para profesionales asesores, particularmente psicólogos que lleven adelante intervenciones en el campo de las organizaciones.

Palabras clave: Aprendizaje organizacional; pensamiento sistémico; sistemas complejos

\section{RESUMO}

Este artigo é desenvolvido no âmbito de uma investigação interessada nas condições envolvidas no desenvolvimento da aprendizagem nas organizações contemporâneas. Com apoio em fundamentos teóricos da aprendizagem organizacional e perspectivas de sistemas complexos e um desenho metodológico de estudos de caso múltiplo, o trabalho enfoca 0 papel do pensamento sistêmico como condição relevante para a aprendizagem organizacional, observada no âmbito dos processos de tratamento e solução de problemas em pequenas e médias organizações na cidade de Córdoba (Argentina). Como resultados, são apresentadas quatro dimensões relevantes da observação em torno do pensamento sistêmico em circunstâncias da prática concreta e a hipótese de que o pensamento sistêmico pode ser apresentado como um padrão auto-organizado. Dentre as conclusões, argumenta-se que 0 pensamento sistêmico tem um impacto significativo nos processos de tratamento de problemas e, consequentemente, na obtenção de melhorias, aprendizado compartilhado, inovações e construção de conhecimento nas organizações estudadas, e que se manifesta como um emergente interacional e competência contextual e não meramente individual. Os resultados têm implicações para pesquisadores, gerentes institucionais e consultores profissionais, principalmente psicólogos que realizam intervenções no campo das organizações.

Palavras-chave: Aprendizagem organizacional; pensamento sistêmico; sistemas complexos

\section{ABSTRACT}

This article is developed within the framework of an investigation interested in the conditions involved in the development of learning in 
contemporary organizations. With support in theoretical foundations of organizational learning and complex systems perspectives and a methodological design of multiple-case studies, this paper focuses on the role of systemic thinking as a relevant condition for organizational learning, observed in the framework of treatment and solution processes of problems in small and medium organizations in Córdoba city (Argentina). As results, we present four relevant dimensions around systemic thinking in circumstances of concrete practice, and the hypothesis that systemic thinking can be presented as a self-organized pattern. As conclusions, it is argued that systemic thinking has a significant impact on the processes of treatment of problems, and consequently on the achievement of improvements, shared learning, innovations and construction of knowledge in the organizations studied, and that it manifests itself as an emerging interactional and contextual rather than as merely individual competence. The findings have implications for researchers, institutional managers and professional advisors, particularly psychologists who carry out interventions in the field of organizations.

Keywords: organizational learning; systemic thinking; complex systems.

n la llamada sociedad del conocimiento la capacidad de aprender e innovar de
las organizaciones constituye un intangible crucial para la generación de valor, la sustentabilidad, el desarrollo de ventajas competitivas, la salud y el bienestar colectivo. Las exigencias actuales de un contexto caracterizado por el cambio permanente ponen en tensión los modos de funcionamiento privilegiados en la modernidad burocrática y desafían a las organizaciones en sus posibilidades de generar el saber, administrar la incertidumbre y gestionar la información disponible.

La búsqueda de comprensiones acerca de cómo aprenden las organizaciones viene siendo tema de investigación y debate en el campo del análisis organizacional y de la administración. La psicología del trabajo y las organizaciones en Latinoamérica no es ajena a estos planteos y la problemática del cambio y el aprendizaje organizacional está presente en sus tópicos de interés y estudio (Santana Cárdenas, 2007).

Sin embargo, el aprendizaje organizacional resulta aún un concepto no acabado y es posible encontrar en la literatura sobre el tema múltiples definiciones e interpretaciones respecto a los elementos, condiciones o variables que lo posibilitan o lo inhiben. 
Trabajos recientes que abordan la problemática en el contexto latinoamericano enfatizan el necesario carácter situado, práctico, agencial e interaccional del conocimiento y del aprendizaje, y señalan de distintas maneras una diversidad de aspectos participantes (Castañeda Zapata, 2015; López Zapata, García Muiña \& García Moreno, 2016).

En una búsqueda de dimensiones relevantes relacionadas con el aprendizaje organizacional, Fassio y Rutty (2017) realizan un trabajo de sistematización de las coincidencias y las organizan en seis grandes temas: (a) aspectos vinculados con la cultura organizacional, (b) la respuesta a la solución de problemas, (c) el liderazgo orientado a la construcción e integración de equipos, (d) el desarrollo de las capacidades del personal y de ambientes de aprendizaje, (e) la comunicación y el libre flujo de la información, y (f) apertura al cambio como oportunidad (p.91). Las autoras señalan además los desafíos que conlleva la dificultad de observación y medición del aprendizaje en la organización, y la escasez de estudios con abordajes cualitativos que incorporen la mirada de los actores más allá de opiniones o aplicación de escalas. Una línea de desarrollo inicialmente planteada en los trabajos de Argyris y Schon (1978) y seguida posteriormente por Senge (1990), ha puesto el foco en los patrones de interacción cognitiva y en las rutinas de abordaje de problemas como fuentes de alta incidencia en las posibilidades de acción inteligente y aprendizaje en la organización. Siguiendo esas posturas, Gore (2003) afirma que cualquier posibilidad de mejora o aprendizaje en la organización está mediada por la calidad de los procesos interaccionales, y que "la mente colectiva es más desarrollada y más capaz de acción inteligente cuanto más ajustada sea la interrelación entre los miembros" (p. 236). En ese sentido insiste en la necesidad de abordar la calidad de los procesos de la mente colectiva, con especial cuidado en la explicitación de las rutinas de abordaje de problemas que van más allá de modelos de aprendizaje individual. 
Para la psicología de las organizaciones la consideración de los procesos mentales ha resultado relevante desde sus inicios, y los aportes de la psicología cognitiva contemporánea han puesto en relieve los fenómenos por los cuales los sujetos reciben, interpretan, almacenan, evalúan y utilizan la información en sus actividades de trabajo (Munduate, 1997; Schein, 1997). Al respecto, y bajo la consideración de la organización como entidad cognitiva, la resolución de problemas y toma de decisiones son estudiados como fenómenos relevantes, destacando en ello la relevancia que adquiere considerar las etapas de reconocimiento y representación del problema, en las que resultan constatables discrepancias de percepción dadas naturalmente por variables del entorno, expectativas o experiencias previas. Asimismo, se señala el carácter de no linealidad de estos procesos y el imbricado interjuego de interacciones existente entre preferencias, contradicciones, ambigüedades, escasez de recursos, sesgos de información, etc.

En ese contexto de antecedentes, llevamos adelante una investigación sobre procesos de cambio y aprendizaje organizacional en pequeñas y medianas organizaciones de la ciudad de Córdoba (Argentina). La investigación asume un interés particular por comprender los procesos de interacción cognitiva dados en el marco de la identificación de problemas cotidianos y su posible transformación en innovaciones de diseño organizacional que garanticen su superación y prevención en el futuro. En ese sentido, la investigación tiene por propósito identificar las condiciones estructurales y dinámicas de la organización que faciliten rutinas de interacción consecuentes con la producción de conocimiento, el aprendizaje e innovación eficaz.

El avance de la investigación que da marco a los elementos presentados en este trabajo, nos ha llevado a identificar situaciones en las cuales los sujetos y los grupos frente a la tarea de resolver problemas, ponen en juego modalidades de comportamiento diferenciadas que oscilan entre manifestaciones de apertura explorativa y manifestaciones de bloqueo defensivo (Montenegro \& Schroeder, 2019a). 
Según lo observado, estas variaciones inciden de manera significativa en el destino que tendrán los intentos de abordaje individual y colectivo de las situaciones problemáticas emergentes en la vida organizacional, ya sea hacia su transformación en innovaciones eficaces o bien, hacia procesos de bloqueo interaccional progresivo. El análisis comparativo de los registros recogidos a lo largo de la investigación nos muestra que en circunstancias en las que las situaciones problemáticas resultan ser transformadas en innovaciones organizacionales eficaces puede reconocerse de manera común y recurrente la participación del pensamiento sistémico como un elemento relevante (Montenegro \& Schroeder, 2019b).

Es así que este artículo presenta resultados de investigación en torno al pensamiento sistémico aplicado, que siendo referido por la literatura sobre el tema, puede ser concebido como una condición relevante para el aprendizaje, la innovación y creación organizacional de conocimiento, así como un recurso significativo para la exploración abierta y generativa en el proceso interaccional de trasformación de problemas en innovaciones organizacionales eficaces.

Desde un análisis basado en principios de la perspectiva constructivista de sistemas complejos (García, 2006; Rodríguez Zoya \& Roggero, 2014) y de la teoría fundamentada (Strauss \& Corbin, 2002), es nuestro objetivo en este trabajo desarrollar una caracterización del pensamiento sistémico aplicado entendido como un componente de la dinámica de interacción cognitiva. En ese sentido, se profundiza en cuatro dimensiones del pensamiento sistémico que el análisis micro interaccional nos ha permitido identificar: (a) configuración del problema (b) abarcabilidad de factores, (c) supuestos de causalidad, y (d) soportes simbólicos apelados. Los resultados obtenidos, por un lado, nos llevan a concebir estas dimensiones en términos de componentes estructurales que explican los fenómenos de estabilidad y cambio del pensamiento sistémico y que median en las posibilidades de su sustentabilidad a nivel de la interacción cognitiva entre los sujetos miembros de la organización. Por otro 
lado, los resultados nos permiten inferir que el pensamiento sistémico puede presentarse a modo de patrón autoorganizado manifestándose como emergente interaccional y contextual más que como competencia meramente individual.

\section{Pensamiento sistémico y aprendizaje organizacional}

El interés por la visión sistémica ha estado presente en la historia del pensamiento humano, incluso en filósofos presocráticos (Garciandíalmaz, 2011), pero es en las últimas décadas, que el pensamiento orientado a abordar la complejidad se desarrolla como un conjunto de principios generales, herramientas y técnicas específicas. Está presente en una diversidad de campos disciplinares como la física, la biología, la ingeniería, la matemática, la administración, la pedagogía, la psicología, y tiene sus bases en diversos enfoques orientados al estudio de la interrelación de fuerzas que forman parte de un proceso común.

El pensamiento sistémico y complejo ha implicado un proceso no solo teórico y académico, sino histórico y social. Responde a nuevas formas de observar, de conocer y comprender el mundo, de explicar e intervenir en las dinámicas complejas de la vida social (Arbeláez Naranjo, 2016).

En el campo organizacional los enfoques sistémicos se ubican en un contexto de investigación y debates teóricos y metodológicos que ha avanzado hacia perspectivas que conciben la organización como entidad cognitiva, como sujeto de aprendizaje y de creación de conocimiento (Bueno Campos, 2004). El pensar de manera sistémica es señalado como recurso necesario para dar respuesta a problemas dinámicamente complejos en consideración de las múltiples situaciones en que individuos, grupos y organizaciones necesitan trascender los hechos como circunstancias aisladas y fragmentadas para pasar a ver las fuerzas e interrelaciones - frecuentemente invisibles y subyacentes - que modelan el cambio y el comportamiento en la organización (Ackoff, 2008; Checkland, 1993; Senge, 1990). 
La relación entre el pensamiento sistémico y el aprendizaje organizacional, planteado inicialmente por los trabajos de Senge (1990), ha sido retomada por diversos estudios. Particularmente, en el contexto latinoamericano, el interés por el aprendizaje organizacional y su relación con el pensamiento sistémico se ve reflejado en trabajos recientes. Gómez Degraves (2012), en una búsqueda de medición de la inteligencia organizacional, define el pensamiento sistémico y la gestión del conocimiento como elementos impulsores del aprendizaje organizacional. Vera y Ospina (2016) destacan la importancia del aprendizaje organizacional como mecanismo de consecución de los objetivos organizacionales y el valor del pensamiento sistémico para evitar o superar errores y lograr visiones compartidas. Muñoz (2017) plantea la relevancia de la formación en pensamiento sistémico por cuanto permite identificar problemas, analizarlos y resolverlos, optimizar los procesos y los sistemas de gestión organizacional, contribuye a la anticipación, al cumplimiento de metas y objetivos, y puede acelerar el aprendizaje organizacional.

\section{La perspectiva de Sistemas complejos}

Resultan relevantes para nuestro estudio, en primer lugar, las perspectivas constructivistas de sistemas complejos, cercanas a los fundamentos del pragmatismo epistemológico (García, 2006; Rodríguez Zoya \& Roggero, 2014) y, complementariamente, un conjunto de fundamentos teóricos que con apoyo en las teorías del caos y la criticalidad autoorganizada se ofrecen desde las denominadas ciencias de la complejidad.

En el proceso de inteligibilización de un sistema complejo es preciso considerar que su estructura está conformada por elementos heterogéneos articulados de manera no descomponible en un marco de diversos niveles de organización. Tales niveles se estructuran bajo principios de autosimilitud e iteración recursiva, de manera que se 
organizan en configuraciones de componentes, que a su vez constituyen componentes de configuraciones de mayor escala (García, 2006; Reynoso, 2006).

A los fines de este artículo, brindan un aporte particular las nociones de estado estable, emergencia, autoorganización, y causalidad circular. La noción de estado tiene un papel relevante en la perspectiva de sistemas complejos, dado el interés central por la problemática de la estabilidad y el cambio de los sistemas. Dicha noción tiene, en dos sentidos al menos, un componente significativamente dinámico. Por un lado, los estados estables son sólo una instancia temporal en la trayectoria de los sistemas, que alterna con instancias de cambios de estructuras; por otro lado, los estados estables involucran en sí mismos una condición dinámica y activa del sistema asociada a la noción de homeostasis (García, 2006; Reynoso, 2006).

El concepto de emergencia, en el sentido que proviene de las aportaciones de la biología y la teoría de sistemas, puede establecerse como un fenómeno de afloramiento de propiedades en niveles más altos de una estructura jerarquizada, que si bien resultan de la interacción de elementos pertenecientes a los niveles inferiores, no son deducibles de las propiedades de estos elementos (García Azconobieta, 2005). La noción de autoorganización ha sido referida como la capacidad que ciertos sistemas alejados del equilibrio tienen para desarrollar de manera espontánea patrones y estructuras ordenadas, sin control o acción manipulatoria externa o interna (Reynoso, 2006), y como "la aparición espontánea de patrones ordenados (...) causados por la interacción recurrente y no lineal entre los componentes del sistema" (García Azconobieta, 2005, p. 18). La interconectividad no lineal o causalidad circular entre los componentes del sistema es una característica sustancial de la autoorganización. 


\section{Metodología}

El presente trabajo se enmarca en una investigación, de enfoque cualitativo, planteada bajo la modalidad de diseño de estudio de casos múltiple. El estudio tiene alcance descriptivo y explicativo. El diseño es de casos múltiples, siguiendo -más que la representatividad - el propósito de explorar y definir el fenómeno y su contexto, así como la búsqueda de comparaciones, regularidades y recurrencias, y una comprensión holística y contextual de los procesos en estudio a los fines de hacer generalizaciones analíticas (Fassio, 2018).

Se trata de tres casos de cambio originados autónomamente por organizaciones que han demandado asistencia técnica profesional. La modalidad de asesoría ha estado orientada al apoyo para el cambio y la mejora interna a nivel de la efectividad de la tarea, el clima de relaciones y el bienestar personal y colectivo. La estrategia general de asesoramiento profesional promueve transiciones culturales y para ello toma como fundamento principios y técnicas del aprendizaje organizacional.

La selección de los casos se ha realizado considerado: la accesibilidad en virtud de los acuerdos de cooperación con el equipo de asistencia técnica, las posibilidades de seguimiento longitudinal de los procesos de cambio y aprendizaje, así como similitudes del proceso de asistencia técnica que incluyen la naturaleza de la demanda y el logro de cambios y aprendizajes efectivamente producidos. En este sentido, los casos considerados presentaron mejoras y cambios significativos respecto a las problemáticas que originaron la demanda, caracterizadas por situaciones estancas de malestar y tensión interpersonal, dificultades funcionales y comunicacionales, y disminución de la eficacia conjunta acompañada de considerables afectaciones en los niveles de confianza mutua entre grupos y sujetos. Otros criterios de selección considerados han sido la trayectoria de la organización, mayor a 5 años de permanencia en el mercado local, y la diversidad de rubros: comercialización (Caso 1), logística y transporte (Caso 2), producción industrial (Caso 3). Las organizaciones en 
estudio recibieron acompañamiento y asesoría profesional en el período Marzo Noviembre de 2018, y las entrevistas fueron realizadas entre Junio y Noviembre de 2018.

\section{Procedimiento}

La indagación empírica se ha realizado mediante el diseño y aplicación de entrevistas semiestructuradas a 12 miembros directivos de las organizaciones estudiadas y a 2 miembros del equipo de la asesoría técnica (AT). Han sido 19 entrevistas en total: 14 individuales (Ei) una a cada directivo y a cada profesional AT; 3 entrevistas grupales con directivos (Eg), y 2 entrevistas mixtas, entre directivos y profesionales AT (Em). Las entrevistas han tenido lugar en momentos de la fase diagnóstica y en las etapas de desarrollo e implementación del proceso de asesoría. Así también en distintas circunstancias del proceso, como situaciones de logro de aprendizajes y mejoras y de impedimento o dificultad emergente. Los logros de aprendizaje organizacional son ponderados en relación con la superación constatable de situaciones problemáticas que dieron lugar a la demanda de asistencia técnica.

El diseño de entrevistas involucra tres tipos de preguntas: preguntas focalizantes, destinadas a la detección de situaciones singularmente significativas desde el punto de vista del entrevistado; preguntas exploratorias, que persiguen la caracterización de eventos, acciones o situaciones circunstancialmente significativas; y re-preguntas de apertura que procuran la identificación de componentes inferenciales tácitos involucrados en la percepción, la acción, o la decisión del entrevistado.

Como resguardo ético y de confidencialidad, se preserva la identidad de las personas entrevistadas, así como las organizaciones a las que pertenecen. 


\section{Participantes}

Doce directivos entre 30 y 65 años de edad (8 varones, 4 mujeres), cada uno de ellos responsables de área o sector con personal a cargo. La selección de los mismos se realizó a partir de un muestreo intencional que contempló como principal criterio de inclusión su participación e implicancia en la demanda inicial y en el proceso de asistencia técnica posterior. Los dos profesionales psicólogos miembros del equipo de asesoría técnica han coordinado y participado activamente de todo el proceso de asesoría en las organizaciones estudiadas.

\section{Análisis de datos}

El análisis de los datos y el desarrollo categorial se llevó a cabo particularmente mediante instancias de codificación, muestreo teórico y comparación constante propios de la teoría fundamentada (Strauss \& Corbin, 2002). Complementariamente, se ha apoyado en la perspectiva constructivista de sistemas complejos (García, 2006; Rodríguez Zoya \& Aguirre, 2011); y en principios de modelización de sistemas complejos (Rodríguez Zoya \& Roggero, 2014).

\section{Resultados}

El análisis nos ha llevado a identificar cuatro dimensiones que caracterizan al pensamiento sistémico como condición relevante para el logro de aprendizajes organizacionales específicos dados en circunstancias de práctica concreta: (a) configuración del problema (b) abarcabilidad de factores, (c) supuestos de causalidad, y (d) soportes simbólicos apelados.

\section{Configuración del problema}

En los registros empíricos se encuentran alusiones en las que los sujetos entrevistados refieren a la calidad del planteo o configuración del problema específico 
a abordar:“El problema estaba mal planteado, no incluía la preocupación de ambos sectores y eso no nos permitía avanzar y encontrar una solución eficaz" (Directivo 3, Ei, Caso 1); "un momento clave fue cuando pusimos las brechas en la pizarra y la pregunta fue ¿cómo podemos hacer para que A y B mejoren al mismo tiempo?" (Directivo 6, Ei, Caso 2, 2018); "la mitad de todo es la construcción del problema y la definición del propósito, en eso cuanto más abarcativo sea el mapeo de las inquietudes de todos es tan vital como la identificación de los criterios de logro que hayan acordado y explicitado" (AT-a, Em, 2018).

Con configuración del problema nos referimos a la calidad de formulación con la que se define una situación problemática para su abordaje y tratamiento. Responde de alguna manera a la necesidad de problematizar la definición del problema como parte del proceso deliberativo señalada por Flores (1997). Los problemas significativos de la vida real, -a diferencia de los problemas de laboratorio o los problemas referidos a medios y fines- se presentan con bajos niveles de estructuración en el sentido de que pueden ser percibidos de diversas maneras por distintas personas y bajo distintas circunstancias. Los problemas no estructurados son aquellos manifiestos en un sentimiento de inquietud, y que resultan de difícil explicitación sin un intento aparente de simplificación. Se tratan de una condición caracterizada por un "sentido de desajuste entre la realidad percibida y una realidad que podría ser" (Checkland, 1993, p.180).

La configuración del problema involucra decisiones que de manera tácita o explícita establecen -entre otras cosas- niveles de inclusión y relevancia respecto a situaciones, propósitos, criterios e inquietudes en juego, así como declaraciones explícitas o implícitas acerca de los horizontes de opción y los factores potencialmente involucrados.

Los esfuerzos por la explicitación de intereses, de preocupaciones prioritarias o criterios relevantes, así como la disponibilidad de datos fehacientes y de toda 
información pertinente que resulte necesaria son referidos por los entrevistados como insumos críticos para un buen planteo del problema y la calidad de su tratamiento posterior.

\section{Abarcabilidad de factores}

En los registros empíricos se encuentran alusiones a la cantidad y calidad de elementos relevantes que participan de alguna manera de la situación problemática abordada: "Ahora mapeamos el problema, es decir, nos esforzamos por hacer análisis más sistémicos, más integrales que abarquen la mayor cantidad de elementos participantes en el problema" (Directivo 9, Ei, Caso 3, 2018); "entonces ya no es una única solución posible, sino muchas buenas ideas que pueden solucionar en conjunto" (Directivo 12,Eg,Caso 3, 2018).

Los profesionales de asistencia técnica entrevistados también aluden a este aspecto:

Cuando el equipo dedica tiempo a explorar factores, variables o elementos diversos que participan de una situación problemática, ya sea un interés afectado, un dato de impacto relevante, consecuencias no deseables o cualquier otro elemento y lo registran de manera compartida, el análisis se vuelve más rico y potente lo cual redunda en soluciones más creativas y eficaces (AT-b, Ei, 2018).

Esta dimensión del pensamiento sistémico alude a la calidad con la que se pone foco sobre los elementos y relaciones que participan en la conformación, fluctuación o cambio potencial de la situación problemática en cuestión, incluyendo diversos niveles de relevancia. Asimismo, a la posibilidad de visibilización de las condiciones operantes en su conjunto.

De acuerdo a Senge (1990) pensar sistémicamente involucra un esfuerzo por comprender con mayor claridad los patrones del sistema en su totalidad y aportar a 
modificarlos, de describir y comprender las fuerzas y las interrelaciones que regulan y modelan el comportamiento del sistema viendo estructuras y procesos en lugar de solo hechos aislados o instantáneas estáticas.

En este sentido, el pensamiento sistémico busca contrarrestar una tendencia naturalizada por el sentido común que tiende a recortar fenómenos, a identificar fuerzas aisladas, a invisibilizar determinados patrones e interconexiones subyacentes y a debatir de manera antagónica entre distintos factores que bien pueden ser cooperantes entre sí.

\section{Supuestos de causalidad}

Los relatos muestran también alusiones a circunstancias en las que perciben diferencias en el modo de registrar las causas operantes, en la generatividad o no de las circunstancias que son apeladas para explicar el origen y permanencia de las situaciones problemáticas abordadas.

En palabras de los entrevistados: "Se trata de pensar más sistémicamente, venciendo la tendencia espontánea a las explicaciones causa - efecto" (Directivo 11, Eg, Caso 3 , 2018); "había momentos en que nos enredábamos en anécdotas, buscando culpas y justificaciones que no nos conducen a nada" (Directivo 10, Em, Caso 3, 2018). Con frecuencia se manifiestan alusiones a cierta facilidad a "personalizar los problemas" y las limitaciones que eso ocasiona: "si nos quedamos con que el problema es alguien, un grupo o un sector nosotros mismos estamos limitando las posibilidades de acción correctiva" (Directivo 2, Ei, Caso 1, 2018); "es recurrente una tendencia natural a buscar culpables o factores unicausales que parcializan el diagnóstico de la situación y conducen a soluciones igualmente parciales" (AT-b, Ei, 2018).

En ese sentido con supuestos de causalidad aludimos a distintas formas con que pueden ser concebidas las relaciones entre las condiciones actuantes en una situación problemática, de cara a la explicación de cómo y por qué ocurren los problemas, así 
como también a la conformación de alternativas de acción convenientes. Cuando el equipo analiza en términos sistémicos las explicaciones emergen centradas en identificar patrones y circuitos sistémicos más que en personas (o grupos) culpables. De esa manera expande el modo de pensar y abordar problemas complejos, aprehendiendo las interrelaciones en vez de concatenaciones lineales de causa efecto, los circuitos de retroalimentación que refuerzan o contrarrestan, los patrones de causalidad recíproca, los procesos que generan cambio y crecimiento, retroceso o equilibrio (Senge, 1990). El pensamiento sistémico se vuelve necesario allí donde quedan expuestas las limitaciones del pensamiento fragmentado, desarticulado, de linealidad causa-efecto o meramente intuitivo para resolver problemas en su complejidad.

El aprendizaje en individuos, grupos y organizaciones se ve facilitado por el trabajo sobre modelos mentales abiertos a nuevas ideas, a la reflexión, al análisis de creencias, decisiones y errores anteriores (Lord, 2015). De acuerdo a Largacha Martínez, Pinzón y León Velásquez (2015) buenos niveles de aprendizaje y participación se dan cuando la organización genera espacios para explorar cosas atípicas y promueve el pensamiento no lineal para considerar propuestas nuevas e innovadoras.

\section{Soportes simbólicos}

En los registros empíricos se advierten referencias al uso de instrumentos y herramientas que cooperan en la sistematicidad del tratamiento de los problemas: "el gráfico de proceso cambia el modo en que se ve, pero también el modo en que se conversa el problema (Directivo 4, Em, Caso 2, 2018); "no había manera de resolverlo hasta que apelaron al apoyo en recursos de vista simultánea, como fue el organigrama, eso ayudó a ver con claridad los canales de decisión que estaban desdibujados" (AT-a, Ei, 2018); "recuerdo cuando graficamos el proceso, eso cambió 
inmediatamente el modo en que estaba viendo el problema, fue muy positivo (...) y desde aquella vez no tratamos un tema sin ubicarlo primero en el proceso en el que ocurre" (Directivo 5, Ei, Caso 2, 2018); "antes todo parecía en una relación lineal causa - efecto"....y usar los diagramas de proceso, tener toda esa información a la vista es muy efectivo" (Directivo 9, Eg, Caso 3, 2018);

Lo que aportó mucho es el gráfico del proceso. Cuando está a la vista de todos eso te ayuda a pensar con más amplitud, porque si no estás viendo siempre sólo una parte, cada uno ve y habla sólo de la parte que conoce y lo que es peor no ve que no ve (Directivo 7, Ei, Caso 2, 2018).

Los soportes simbólicos aluden a los tipos de recursos representacionales por los cuales una determinada realidad significativa o una circunstancia problemática es registrada y comunicada conjuntamente con sus condiciones de ocurrencia y variación. Los soportes simbólicos permiten ver los problemas en contexto y las relaciones dinámicas (no fácilmente visibles ni concientes) de las fuerzas que operan reforzando o inhibiendo los problemas cotidianos (Senge, 1990).

Las narraciones, los diagramas de proceso o flujogramas, los organigramas, las imágenes, los informes, los procedimientos, son algunos ejemplos de tales recursos apelados de manera diversa en la vida organizacional para configurar las circunstancias significativas. Cada uno de estos tipos de recursos conlleva determinados modos de recortar, configurar y estructurar los elementos y relaciones que hacen a la significación de la circunstancia problemática. En tal sentido, son internalizados y significados de diversa manera por los sujetos a los fines de saber a qué atenerse, de regular sus interacciones posibles, y de organizar su propia experiencia subjetiva en relación con las circunstancias.

Por razones de espacio y para no exceder los límites del escrito, los extractos del relato de los entrevistados se han presentado para cada dimensión a modo ilustrativo. 


\section{Discusión}

El análisis de las circunstancias referidas en los casos en estudio según fundamentos de sistemas complejos, permite plantear como hipótesis que existirían relaciones de interdependencia e implicancia recíproca entre las dimensiones del pensamiento sistémico detalladas, es decir, entre la configuración del problema, la abarcabilidad de factores considerados en su abordaje y tratamiento, los supuestos causales predominantes y los soportes simbólicos apelados. Esto lleva a pensar que la comprensión entitaria de cada una de estas dimensiones no podría realizarse a través de su estudio por separado o de manera aislada. Por el contrario, y aun considerando su naturaleza diversa, tales dimensiones resultarían no descomponibles entre sí a los fines de la identificación de causalidades y propiedades emergentes, e interdefinibles de manera recíproca en el marco de sus contextos específicos de acoplamiento (García, 2006).

Vale decir entonces que el pensamiento sistémico constituye una manifestación específica que la modalidad de pensamiento puede asumir en el proceso deliberativo 0 de tratamiento de problemas, que puede ser interpretada como patrón autoorganizado emergente (Reynoso, 2006) de un conjunto de condiciones de micro nivel conformado por las dimensiones detalladas. Es así que el pensamiento sistémico- diferenciado del pensamiento no sistémico de tipo narrativo o lineal - requiere como condiciones de emergencia y estabilidad (García Azconobieta, 2005) una cierta calidad metódica en la construcción inicial del problema y en el relevamiento sistemático que los sujetos puedan hacer de los factores que inciden en su aparición y/o persistencia; cierto nivel de conciencia en los supuestos de causalidad a los que se apela para el diagnóstico y análisis de la situación problemática, y rigurosidad en los soportes simbólicos utilizados para la representación integral del problema en su contexto.

Ahora bien, desde una perspectiva de observación a mayor escala (García, 2006; Reynoso, 2006), el análisis interpretativo permite inferir que el pensamiento sistémico 
no emerge ni se constituye de manera aislada sino en íntima relación con otros elementos de naturaleza comunicacional, decisional y emocional que participan en la dinámica de micro interacción organizacional. En ese sentido, en la voz de los entrevistados la posibilidad del pensar sistémico aparece vinculado a los modos de conversar: "para tratar los problemas sistémicamente también es importante el modo en el que hablamos, en el que nos escuchamos mutuamente" (Directivo 1, Ei, Caso 1, 2018); "si no mantenemos un diálogo abierto, exploratorio y ordenado no es posible abordar los problemas de manera eficaz" (Directivo 8, Ei, Caso 3, 2018); "vemos en los casos que los cambios hacia un pensamiento más sistémico están asociados a cambios correlativos y alineados al modo de conversar" (AT-a, Em, 2018).

La confianza y buena disposición anímica y emocional también son referidos como aspectos de valor a la hora de tratar los problemas de manera conjunta: "si no confiamos, no podemos hablar con claridad, y mucho menos resolver bien los problemas" (Directivo 3, Ei, Caso 1, 2018); "la confianza es algo que cuesta construir pero una vez que está, perdemos el miedo a hablar de los errores“ (Directivo 5, Eg, Caso 2, 2018); "la predisposición es muy importante, no es lo mismo intentar resolver y decidir sobre los problemas desde una actitud abierta que desde el rencor o el desgano, todo eso suma al clima que colabora también al buen tratamiento" (Directivo 7, Eg, Caso 2, 2018); "con frecuencia vemos que las disposiciones personales y el clima de relaciones se ve favorecido en la medida que la gente cambia su modo de pensar y tratar los problemas" (AT-b, Ei, 2018).

De igual manera la claridad de involucramiento en el proceso decisorio: "pensar los problemas de esta manera vuelve más claro por ejemplo qué es posible delegar" (Directivo 12, Ei, Caso 3, 2018); "el uso de los soportes gráficos en el tratamiento sistémico de situaciones siempre ordena las incumbencias decisionales sobre los distintos temas, en qué cosas corresponde autonomía, qué se consensua y qué temas 
requieren de consulta" (AT-a, Em, 2018); "muchas veces los canales de decisión están desdibujados y eso atenta al buen tratamiento" (AT-a, Ei, 2018).

El análisis de los datos obtenidos nos permite inferir entonces que el pensamiento sistémico emerge y se sostiene en cercana asociación recíproca con actitudes y disposiciones anímicas de apertura y confianza, con reglas explícitas de involucramiento decisional y con modalidades conversacionales de carácter exploratorio y diálogo abierto.

Si bien este punto merece seguir siendo investigado, dicho conjunto de aspectos interactuantes -cierta calidad conversacional, disposición anímica de apertura, claridad de involucramiento decisional y pensamiento sistémico- podría asociarse a una dinámica de sinergia cognitiva en la cual la superación colectiva de los problemas y su transformación en aprendizajes e innovaciones organizacionales eficaces resultarían mayormente posibles. En contraparte, el pensamiento sistémico no parece tener posibilidad de emergencia y estabilidad en condiciones de clausura y bloqueo interaccional defensivo cristalizado en una dinámica de reforzamiento recíproco entre rutinas de conversación pobre, simulatoria o confrontativa, de decisiones unilaterales implícitas o pautas tácitas de involucramiento decisional, de disposiciones emocionales y anímicas asociadas al resentimiento o la resignación, de actitudes negativas de cierre, indiferencia o mero acatamiento, y la apelación a modalidades de pensamiento predominantemente narrativo o de tipo lineal.

En esa línea, cabe comprender a la acción representacional y de pensamiento como fenómeno emergente de la dinámica de interacción cognitiva puesta en juego por parte de los sujetos ante las situaciones problemáticas más allá de su carácter comportamental individual. En consecuencia, la posibilidad de implementación de modalidades sistémicas de pensamiento en el abordaje de los problemas emergentes en la organización está sujeta a condiciones situacionales y contextuales que trascienden los perfiles de disposición o competencia individual. Ello permite 
comprender, por ejemplo, cómo las estrategias de capacitación "áulicas", centradas en la formación en habilidades individuales no resultan por sí mismas condición de implementación efectiva, en relación con el peso que adquieren las rutinas de interacción cognitiva instituidas y emergentes en el ámbito de la acción cotidiana.

Así entendida, esta dinámica de interacción cognitiva puede pensarse como elemento constitutivo de una cultura organizacional orientada al aprendizaje, pero que no ocurre de manera espontánea ni por la voluntad individual o grupal, sino que requiere de políticas institucionales, procedimientos sistemáticos e instrumentos de identificación y gestión activa de las situaciones problemáticas emergentes.

\section{Conclusiones}

En línea con lo que buena parte de la literatura sobre el aprendizaje organizacional señala, el pensamiento sistémico es referido por los participantes del estudio como un elemento de valor, que incide de manera significativa en los procesos de tratamiento eficaz de problemas, y con ello en el logro de mejoras, de aprendizajes compartidos, de innovaciones y de construcción de conocimiento colectivo.

Al respecto, entendemos que resulta oportuno avanzar en el estudio del pensamiento sistémico en las condiciones concretas de la vida social y en un marco de intentos de cambio y transformación. En otras palabras, pasar del pensamiento sistémico como deber ser, al poder ser del pensamiento sistémico aplicado en la práctica.

Creemos que los hallazgos del estudio tienen implicancias para investigadores, para gestores institucionales y para profesionales asesores. A los primeros invita a investigar el comportamiento de las dimensiones del pensamiento sistémico desarrolladas en este trabajo en otros entornos organizativos. También a explorar otras dimensiones posibles.

A directivos y gestores institucionales los resultados de la investigación pueden resultar un aporte y ayuda para resguardar una metodología del pensamiento 
sistémico aplicado fundada en las dimensiones desarrolladas en este trabajo. Pueden servir también de orientación para focalizar sus esfuerzos y recursos en las condiciones que mostraron relación con las posibilidades de emergencia y sostenimiento del pensamiento sistémico, entre ellas el fortalecer prácticas orientadas a generar condiciones de confianza, reglas claras de involucramiento decisional y de diálogo abierto propicio para compartir saberes, conocimientos y experiencias.

Para los asesores técnicos, y en particular a los psicólogos que intervienen en este campo, pretende ser un aporte a las prácticas de cambio, innovación y aprendizaje en las organizaciones, buscando enriquecer el diseño y la gestión de experiencias de asistencia técnica para el aprovechamiento adecuado de esfuerzos económicos y humanos, y la generación de intervenciones sistémicas e integrales que permitan abarcar la complejidad de los aspectos en juego, sin perder de vista el bienestar, la salud y desarrollo de las personas que las integran.

En términos metodológicos, entendemos que ha resultado pertinente al estudio apelar a recursos de racionalidad ampliada desde los cuales las entrevistas se han desarrollado como instancias de reflexividad sistemática guiada permitiendo explorar más allá de las percepciones y opiniones de los entrevistados-, sus saberes y sentidos tácitos y avanzar de manera intersubjetiva en el mapeo de las condiciones participantes.

Finalmente, corresponde aludir a algunas limitaciones del estudio relacionadas con la proveniencia de los registros empíricos, en tanto refieren a miembros directivos de los casos que, si bien acontecen en organizaciones diversas, comparten el enfoque de asesoramiento profesional recibido. Dada la perspectiva de investigación adoptada, el relevamiento de antecedentes investigativos sobre las unidades conceptuales involucradas, lejos está de ser agotado. También nos concierne señalar que el conjunto de proposiciones aquí presentadas guardan ciertos márgenes de provisionalidad y son postuladas en el marco de un proceso de aproximaciones 
sucesivas hacia la inteligibilización de la organización como sistema cognitivo complejo.

\section{Referencias}

Ackoff, R. (2008). Definiendo "sistema". En E. Herrscher, Pensamiento sistémico. Caminar el cambio o cambiar el camino (pp. 217-219). Buenos Aires: Granica.

Arbeláez Naranjo, L. (2016). Pensamiento sistémico y psicología. Un cambio de paradigma para la comprensión de realidades complejas. Revista Poiésis, 296305. doi:10.21501/16920945.2120

Argyris, C.,\&Schon, D. (1978). Organizacional Learning. A theory of action perspective. Massachussets: Addison.

Bueno Campos, E. (2004). Fundamentos epistemológicos de dirección del conocimiento organizativo: desarrollo, medición y gestión de intangibles. $\begin{array}{llll}\text { Economía } \quad \text { (35), } & \text { 13-26. }\end{array}$ dehttp://mincotur.gob.es/Publicaciones/Publicacionesperiodicas/Economialndus trial/RevistaEconomialndustrial/357/03_EduardoBueno_357.pdf

Castañeda Zapata, D. (2015). Condiciones para el aprendizaje organizacional. Estudios Gerenciales, 31, 62-67. doi:10.1016/j.estger.2014.09.003

Checkland, P. (1993). Pensamiento de sistemas, prácticas de sistemas. México: Grupo Noriega.

Fassio, A. (2018). Reflexiones acerca de la metodología cualitativa para el estudio de las organizaciones. Ciencias Administrativas, 6, 73-84. doi:10.24215/23143738e028

Fassio, A.,\&Rutty, M. G. (2017). Hacia la identificación de dimensiones relevantes relacionadas con el aprendizaje organizacional. Trilogía Ciencia Tecnología Sociedad, 9(16), 85- 99.doi:10.22430/21457778.179

Flores, F. (1997). Inventando la empresa del siglo XXI. Santiago de Chile: Granica. 
García Azconobieta, T. (2005). Evolución, desarrollo y (auto) organización. Un estudio sobre los principios filosóficos de la evo-devo. Donostia- San Sebastián: Universidad del País Vasco.

García, R. (2006). Sistemas complejos. Conceptos, método y fundamentación epistemológica de la investigación interdisciplinaria. Barcelona: Gedisa.

Garciandíalmaz, J. A. (2011). Pensar sistémico: una introducción al pensamiento sistémico. Bogotá, Colombia: Pontificia Universidad Javeriana.

Gómez Degraves A. (2012). Propuesta metodológico-estadística para medir la inteligencia organizacional, fundamentada en la Quinta Disciplina de Peter Senge. $\quad$ Negotium, $\quad$ 22(9), 53-83. Recuperado dehttp://revistanegotium.org.ve/pdf/22/art3.pdf

Gore, E. (2003). Conocimiento Colectivo. Granica: Buenos Aires.

Largacha Martínez, C., Pinzón, A., \& León Velásquez, E. (2015). La fusión de la gerencia humanista y el aprendizaje organizacional producen organizaciones sostenibles y de excelencia. Revista Escuela De Administración De Negocios, (78), 74-91. doi:10.21158/01208160.n78.2015.1191

López Zapata, E., García Muiña, F.,\& García Moreno, S. (2016). Atributos de la organización que aprende: una revisión de la literatura. Revista Internacional de Organizaciones, 16, 59-81. doi:10.17345/rio16.59-81

Lord, M. (2015). Group learning capacity: the role of open-mindedness and shared vision. Frontiers in Psychology, 6. doi:10.3389/fpsyg.2015.00150

Montenegro, G., \& Schroeder, I. (2019a). El estado de Bloqueo Difuso Generalizado. Un obstáculo interaccional a los procesos de aprendizaje en la organización. Debates actuales en Psicología y Sociedad, 2(2), 30-52. doi: 10.22529/daps.2019.2(2)03

Montenegro, G., \& Schroeder, I. (2019b). Procesos de Aprendizaje Organizacional. Un análisis interpretativo desde la perspectiva de Sistemas Complejos. Gestión y 
$\begin{array}{llll}\text { Estrategia, } & 56, & 9-22 . & \text { Recuperado }\end{array}$ dehttp://gestionyestrategia.azc.uam.mx/index.php/rge/article/view/669/600

Munduate, J. (1997). Psicología social de la organización. Las personas organizando. Madrid: Pirámide.

Muñoz, L. (2017). Propuesta para el desarrollo del pensamiento sistémico en los programas de formación de posgrado del convenio USTA-ICONTEC. Signos, 9(2), 121-148. doi:10.15332/s2145-1389.2017.0002.08

Reynoso, C. (2006). Complejidad y el caos: Una exploración antropológica. Buenos Aires: Sb.

Rodríguez Zoya, L., \& Aguirre, J. (2011). Teorías de la complejidad y Ciencias Sociales. Nuevas estrategias epistemológicas y metodológicas. Nómadas. Critical Journal of Social and Juridical Sciences, 30(2). Recuperado dehttp://www.redalyc.org/articulo.oa?id=18120143010

Rodríguez Zoya, L., \& Roggero, P. (2014). La modelización y simulación computacional como metodología de investigación social. Polis, 13(39), 417440. Recuperado de https://scielo.conicyt.cl/scielo.php?pid=S071865682014000300019\&script=sci_abstract\&tIng=en

Santana Cárdenas, S. (2007) Psicología del trabajo y de las organizaciones: estado del arte, retos y desafíos en América Latina. Revista Perspectivas en Psicología, $\quad 25-38 . \quad$ Recuperado de https://www.researchgate.net/publication/309417860_Psicologia_del_trabajo_y _de_las_organizaciones_estado_del_arte_retos_y_desafios_en_America_Latin a

Schein, E. (1997). Psicología de la organización. México: Prentice Hall.

Senge, P. (1990). La quinta disciplina. Buenos Aires: Granica. 
Strauss A., \& Corbin, J. (2002). Bases de la investigación cualitativa. Técnicas y procedimientos para desarrollar la teoría fundamentada. Bogotá: Universidad de Antioquia.

Vera, M.,\& Ospina, Y. (2016). Importancia del aprendizaje organizacional como mecanismo que favorece la consecución de los objetivos organizacionales. Colección Académica de Ciencias Sociales, 3(1), 10-41. Recuperado de https://revistas.upb.edu.co/index.php/cienciassociales/article/view/6190

\section{Declaración de contribución de los autores:}

Este artículo de investigación científica y tecnológica es parte de una línea de investigación sobre aprendizaje organizacional, cambio e innovación en organizaciones desde la cognición distribuida y la perspectiva de sistemas complejos. La investigación está financiada por la Universidad de Católica de Córdoba, y radicada en la Facultad de Filosofía y Humanidades y en el Instituto de Ciencias de la Administración (ICDA).

SGM e IKS son el director y co-directora de la investigación respectivamente, ambos comparten la responsabilidad en todo el proceso de escritura, revisión y versión final del manuscrito.

\section{Editor se sección}

La editora de sección de este artículo fue Karina Curione.

ORCID ID: 0000-0002-4069-5615 
Formato de citación

Montenegro, G. y Schroeder, I. (2020). Dimensiones del pensamiento sistémico aplicado: un estudio de casos múltiple desde la perspectiva de sistemas complejos y el aprendizaje organizacional. Psicología, Conocimiento y Sociedad, 10(2), 74-100. doi: http://dx.doi.org/10.26864/PCS.v10.n2.4 